\title{
Evaluación de la fuerza de adhesión de un sistema adhesivo a la superficie de esmalte blanqueado con peróxido de carbamida al $10 \%$ con fluor y sin fluor
}

Evaluation of an adhesive system bond strength to the surface of bleached enamel with $10 \%$ carbamide peroxide with and without fluoride

\section{Resumen}

El objetivo del presente estudio in vitro fue evaluar la fuerza de adhesión, de un sistema adhesivo, a la superficie de esmalte blanqueado con peroxido de carbamida al $10 \%$ con fluor y sin fluor incorporado. Con este fin se emplearon nueve terceras molares sanas, las cuales fueron divididas en tres grupos: grupo I: blanqueado con Opalescence al 10\% PF (con Fluor), grupo II: blanqueado con Opalescence 10\% (sin fluor), grupo III: grupo control sin blanqueamiento. Los grupos de blanqueamiento pasaron por un periodo de blanqueamiento de 6 horas por día a una temperatura de $37^{\circ} \mathrm{C}$ durante 14 días consecutivos y fueron almacenados en saliva artificial.

Se esperó 7 días para la colocación de un bloque de resina (Filtek Z350, 3M) de 5 × 5 x $3 \mathrm{~mm}$. (anchura, longitud, altura) colocada en la superficie bucal, palatina o lingual de cada diente, previamente se uso ácido fosforico al 35\% (Scotchbond, 3M) y el Sistema Adhesivo (Single Bond 2, 3M). Luego mediante el uso de un disco diamantado biactivo se realizaron cortes horizontales y verticales para obtener los especimenes, los cuales fueron almacenados en agua bidestilada. Luego de 24 horas los especimenes fueron examinados con el método de microtensiometro a $0,5 \mathrm{~mm} / \mathrm{min}$ obteniéndose los valores de fuerza de adhesión. Los datos fueron analizados con el test ANOVA y post test de Tukey para determinar las diferencias significativas entre los grupos experimentales $(p<0.05)$.

Los resultados obtenidos fueron: grupo I $=28.47 \pm 5.89 \mathrm{MPa}$, grupo II $=24.51 \pm 7.96 \mathrm{MPa}, \mathrm{y}$ grupo control $=30.51 \pm 7.48 \mathrm{MPa}$. La fuerza de adhesión del sistema adhesivo en el grupo con superficie, de esmalte blanqueado con peroxido de carbamida $10 \%$ y con $0.11 \%$ de ion fluoruro fue significativamente mayor que en el grupo cuya superficie del esmalte fue blanqueado con peroxido de carbamida $10 \%$ sin fluor incorporado. Se concluyó que bajo las condiciones de éste estudio la incorporación del ion fluor al $0.11 \%$ en el peroxido de carbamida 10\% (Opalescente), preserva la fuerza de adhesión a esmalte, en comparación con el peroxido de carbamida $10 \%$ que no contiene fluor en su composición (Opalescente $\mathrm{PF})$ después de un régimen de blanqueamiento.

\footnotetext{
Abstract

The purpose of this study in vitro was to evaluate the tensille strength of adhesive system to the surface of bleached enamel with $10 \%$ carbamide peroxide with and without fluoride. We used nine healtly third molars, divided into three groups: Control (unbleached), group I (bleached with 10\% Opalescence), group II (bleached with 10\% Opalescence PF). Bleaching groups had a period of bleaching of 6 hours per day at $37^{\circ} \mathrm{C}$, during 14 consecutive days; the teeth during this period were stored in artificial saliva.

We waited 7 days to put a resin block (Filtek Z350,3M) of $5 \times 5 \times 3 \mathrm{~mm}(\mathrm{w}, \mathrm{l}, \mathrm{h})$ on the buccal, palate or lingual surface of each tooth, previously we used $35 \%$ phosphoric acid (Scothbond, $3 \mathrm{M}$ ) and an adhesive system (Single Bond 2,3M). We used a diamond bioactive disc to make a horizontal and vertical cut to obtain the specimens, which were stored in distilled water. After 24 hours, the specimens were tested with the microtensile method at $0.5 \mathrm{~mm} / \mathrm{min}$. Data were analyzed with ANOVA and post Tukey tests to determine significant differences between the experimental groups $(\mathrm{p}<0.05)$.

The results were: Control group $=30.51 \pm 7.48 \mathrm{MPa}$, group $\mathrm{I}=24.51 \pm 7.96 \mathrm{MPa}$, group $\mathrm{II}=28.47 \pm$ $5.89 \mathrm{MPa}$. The bond strength of adhesive system to the surface of bleached enamel with $10 \%$ carbamide peroxide with $0.11 \%$ fluoride ion is more significant than the surface of bleached enamel with $10 \%$ carbamide peroxide without fluoride.

Under conditions of this study, we concluded that the addition of fluoride ion in the carbamide peroxide (Opalescence) preserves the bond strength in comparison with carbamide peroxide that does not contain fluoride in its composition (Opalescence PF), after a bleaching period.
}

\section{Introducción}

A través de la historia, los criterios de estética han sido influenciados por la cultura y el ambiente; por ejemplo, una referencia del año 2000 a.c. menciona la costumbre japonesa de decolorarse los dientes, este procedimiento se denominaba "Ohaguro", cuyo resultado era una sonrisa con dientes negros o marrones oscuros, en el apogeo de la civilización Maya los dientes desgastados eran

\section{Artículo Original}

\section{Jocelyn Graciela Lugo Varillas Hernán Horna Palomino ${ }^{2}$}

CD Práctica Privada.

Dpto Academico Estomatología Rehabilitadora. Facultad Odontología. UNMSM. Lima - Perú.

\section{Correspondencia:}

C.D Jocelyn Graciela Lugo Varillas Av. Brasil 2481-B Of. 205 Jesús María. Lima - Perú

Tlf: 6197000 / 3403

e-mail: jocelynlugo@hotmail.com

Palabras Clave: Peróxido de carbamida fluorado, esmalte dental, fuerza de adhesión.
Key words: Carbamide peroxide. Fluoride Tooth enamel. Bonding strength adornados con incrustaciones de piedras nefríticas ${ }^{(1)}$; otras citas relatan que durante la España preromana, los sumos sacerdotes manifestaban que enjuangarse los dientes con orina mantenían los dientes saludables y blancos. 
En el mundo moderno dientes blancos, bien contorneados y alineados establecen el patrón de belleza, pero no son solo considerados partes del atractivo; también son indicadores de salud nutricional, autoestima, higiene, nivel económico y sexualidad.

El blanqueamiento dental es uno de los procedimientos más solicitados de la odontología estética, es un procedimiento que elimina manchas o coloraciones de origen extrínseco o intrínseco; a pesar de considerarse un procedimiento conservador presenta efectos adversos que hay que tener en consideración.

Existen muchos estudios in vitro sobre los efectos adversos del blanqueamiento en el esmalte, investigaciones en microscopia electrónica del esmalte blanqueado que presentaron poco o nada de alteraciones y otros reportaron cambios significativos en la superficie de esmalte, como aumento en la porosidad, erosión y desmineralización; también alteraciones en la composición química, en las propiedades mecánicas como microdureza y fuerza de adhesión en relación con los materiales dentales.

Existe varias técnicas de blanqueamiento una de las menos invasivas es la técnica del blanqueamiento supervisada por el profesional y administrado por el mismo paciente usando cubetas de acetato y utilizando como agente blanqueador el peróxido de carbamida al 10\%, esta concentración mínima es segura sobre todo para tejidos blandos y es considerada un método efectivo para el blanqueamiento en dientes vitales ${ }^{(2)}$.

Debido que en el mercado existen diversos agentes blanqueadores por la gran demanda de tratamientos estéticos, uno de los cuales son los agentes blanqueadores fluorados, en estos últimos años han aparecido algunos estudios donde evalúan estos agentes blanqueadores con contenido de flúor, como el estudio relacionado con el potencial de remineralización de un sistema blanqueador de peróxido de carbamida $10 \%$ con flúor sobre el esmalte previamente desmineralizado, concluyendo que la adición del flúor a un sistema blanqueador dental no afecta la eficacia de este gel blanqueador y mejora las propiedades de remineralización ${ }^{(3)}$, en este estudio la superficie del esmalte dental al captar el ión flúoruro incorporado en el mismo blanqueador estaría incrementando la resistencia del esmalte a la desmineralización, esto provocaría una mínima alteración de la estructura morfológica del esmalte por tanto ayudaría a mejorar la fuerza de adhesión.

Sobre la fuerza de adhesión en un esmalte tratado con un blanqueador fluorado, existe un estudio en el que los agentes blanqueadores de peroxido de carbamida $10 \%$ que contienen calcio o flúor, este último en concentraciones de 0,5 y $0,2 \%$ de ión fluoruro, presentaron una similar fuerza de tensión que el grupo control, el cual no fue blanqueado, se demostró que el tratamiento de blanqueamiento con agentes blanqueadores en base de peróxido de carbamida $10 \%$ que contienen iones de fluoruro o calcio no cambian la ultima fuerza de tensión del esmalte humano ${ }^{(4)}$.

Por todo ello el propósito del estudio es evaluar la fuerza de adhesión de un sistema adhesivo a la superficie de esmalte blanqueado con peróxido de carbamida $10 \%$ con flúor $(0,11 \%$ ión fluoruro) y sin flúor, colocada después de una semana del blanqueamiento, tiempo mínimo que es recomendado para realizar una restauración estéti$\mathrm{ca}^{(5)}$, usando productos que tengan mayor demanda en el mercado.

\section{Materiales y Método}

\section{La Muestra:}

Se seleccionaron 9 terceras molares humanas extraídas de pacientes de 18 a 25 años, que cumplieran los siguientes criterios de inclusión:

- Extraídas por razones ortodónticas, periodontales o por razones protésicas.

- Ápice cerrado

- Sin caries

- Sin líneas de fractura en el esmalte

- Sin manchas blancas.

Los grupos se conformaron en forma aleatoria: 3 grupos obteniendo un total de 99 especimenes, de los cuales 33 especimenes corresponde al grupo control: no blanqueado, 33 especimenes al grupo I: blanqueado con peróxido de carbamida $10 \%$ sin flúor y 33 especimenes al grupo II: blanqueado con peróxido de carbamida $10 \%$ con flúor.

\section{Obtención de la muestra}

Se seleccionó 9 terceras molares sanas, con ápice cerrado, la edad del paciente fluctuó entre los 18 a 25 años.

Las piezas se almacenaron en un recipiente de vidrio, con suero fisiológico, bajo refrigeración a $-4^{\circ} \mathrm{C}$; según la $\mathrm{ISO}^{(6)}$ solo se debe de almacenar por un máximo de seis meses luego de la extracción.

\section{Preparación de la muestra:}

Se confeccionó 3 bases de acrílico, en donde se colocaron los dientes, para ello primero se construyó bases de cera, luego se tomó impresiones de éstas y se hizo un vaciado con acrílico de termocurado.

Se formaron en forma aleatoria 3 grupos experimentales:

- GrupoI:serealizóel blanqueamiento con peroxido de carbamida al $10 \%$ sin contenido de Flúor (Opalescence)

- Grupo II: se realizó el blanqueamiento con peroxido de carbamida al $10 \%$ con contenido de Fluor al $0,11 \%$ y nitrato de potasio (Opalescence PF)

- Grupo control: no se realizó el blanqueamiento

Para confeccionar las cubetas de acetato se prepararon modelos de yeso piedra de las arcadas, se aliviaron y se confeccionó las cubetas de acetato de espesor de 0,19 $\mathrm{mm}$ en una máquina de vacuo.

\section{Procedimiento de blanqueamiento}

Antes de proceder con el blanqueamiento se realizó un corte en los dientes a $3 \mathrm{~mm}$ debajo de la furca con un disco diamantado de doble cara activa (KG-Sorensen, Brasil) y se procedió a retirar el tejido pulpar con tiranervios y lima N 20 (Maillefer, swiss), la cámara pulpar fue lavada con agua bidestilada; luego se preparó la pieza dental desgastando $0,5 \mathrm{~mm}$ aproximadamente del esmalte con discos soflex en la cara vestibular y palatina o lingual, este desgaste se realizó con la finalidad de obtener una superficie plana la cual ayuda en el cálculo del área adhesiva y en el test de microtension; este desgaste se realizó perpendicular al piso y con una irrigación constante con agua bidestilada, se hizo una previa limpieza del esmalte con una pasta de piedra pómez tamizada con una escobilla de profilaxis; después al grupo I se realizó el blanqueamiento con peróxido de carbamida al 10\% (Opalescence) sin 
flúor y al grupo II con peróxido de carbamida al $10 \%$ con contenido de fluor al 0,11\% (Opalescence PF) por 6 horas diarias durante 14 días, almacenando los dientes durante los días de blanqueamiento en saliva artificial (1g sodium carboxymethylcellulose, 4,3g xylitol, 0,1 $\mathrm{gCl}, 0,1 \mathrm{~g} \mathrm{NaCl}, 0,02$ $\mathrm{mg} \mathrm{NaF}, 5 \mathrm{mg} \mathrm{MgCl}_{2}, 5 \mathrm{mg} \mathrm{CaCl}{ }_{2}, 40 \mathrm{mg}$ $\mathrm{K}_{2} \mathrm{HPO}_{4} 1 \mathrm{mg}$ potasium thiocynate $\mathrm{y}$ $100 \mathrm{mg}$ distilled deionized water) a $37^{\circ} \mathrm{C}$, la saliva artificial fue cambiada cada 3 días.

\section{Procedimiento de adhesivo}

Luego de una semana del blanqueamiento se procedió a la colocación de la resina (Filtek 350, 3M), para lo cual se cortó el diente por la mitad en sentido mesiodistal, seguidamente se procedió al grabado ácido de la zona de esmalte con ácido fosfórico al 35\% (scothbond) por 15 segundos en la superficie vestibular y palatina o lingual, para luego colocar el adhesivo (Single bond 2) y después colocar la resina (filtek Z 350) construyendo de ésta manera un cubo de resina de $5 \times 5 \mathrm{~mm}$ y una altura de $3 \mathrm{~mm}$ aproximadamente, a través de una aplicación secuencial de 1 a $2 \mathrm{~mm}$ de capas del material, cada una fotocurada por 40 segundos; seguidamente se colocó resina en el lado opuesto de la pieza $2 \mathrm{~mm}$ de altura para servir de soporte. Todas las piezas dentales fueron almacenadas en saliva artificial 24 horas a $37^{\circ} \mathrm{C}$.

\section{Obtención de los especímenes}

24 horas después todas las piezas fueron fijadas en un bloque de acrílico

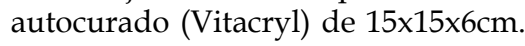
Se realizó los cortes con la máquina de corte isomed (Buehler Ltd., Lake Bluff, IL), estos cortes fueron perpendiculares a la superficie de adhesión en forma horizontal y vertical, 4 cortes horizontales y 4 verticales; obteniendo especimenes de $10 \times 0.8 \times 0.8 \mathrm{~mm}$ aproximadamente, los cuales fueron almacenados en agua bidestilada a temperatura ambiente por 24 horas.

De acuerdo a la $\mathrm{ISO}^{(6)}$ la prueba de microtensión se puede realizar después de 24 horas de haber hecho los cortes de los especimenes, tiempo suficiente para discriminar entre aquellos materiales que no resisten un ambiente húmedo.

\section{Test de microtensión}

Los especimenes fueron fijados cuidadosamente en el dispositivo de prueba con cianoacrylato Zapit (Dental Ventures of América, Corona, CA) y analizados en la máquina de microtensión (Bisco, Schaumburg, IL) con

una fuerza constante a la velocidad de $0,5 \mathrm{~mm} / \mathrm{min}$ hasta observar la fractura del mismo.

\section{Resultados}

Para comparar la medias de la fuerza de adhesión entre los grupos de

\section{Cuadro1. Fuerza de adhesión de los grupos experimentales en MPa}

\begin{tabular}{lcccc}
\hline $\begin{array}{c}\text { grupos } \\
\text { experimentales }\end{array}$ & No & Media & $\begin{array}{c}\text { Desviación } \\
\text { Standard }\end{array}$ & Rango \\
\hline PC10\% s/F & 33 & 24,51 & 7,96 & 26,54 \\
PC10\% c/F & 33 & 28,47 & 5,89 & 22,60 \\
Control & 33 & 30,51 & 7,48 & 30,37 \\
\hline
\end{tabular}

F intergrupos $=6.701, p=0.002$

El análisis post hoc mediante el test de Tukey (cuadro N 03) confirma que el blanqueamiento con peróxido de carbamida $10 \%$ sin flúor a la superficie de esmalte produce una fuerza de adhesión significativamente menor comparándola con el grupo control $(\mathrm{p}=0,01)$.

Del mismo modo se confirma que el blanqueamiento con peróxido de carbamida $10 \%$ con flúor a la superficie de esmalte produce una fuerza de adhesión significativamente mayor comparándola con un blanqueamiento con peróxido de carbamida $10 \%$ sin flúor a la superficie del esmalte $(p=0,047)$

También se confirma que el blanqueamiento con peróxido de carbamida $10 \%$ con flúor a la superficie de esmalte no produce una fuerza de adhesión significativa comparándola con el grupo control $(p=0,466)$.

\section{Cuadro 3. Prueba de Tukey}

\begin{tabular}{llcc}
\hline \multicolumn{1}{c}{$\begin{array}{c}\text { grupos } \\
\text { experimentales }\end{array}$} & $\begin{array}{l}\text { diferencia } \\
\text { de medias }\end{array}$ & error típico & significancia \\
\hline PC10\% s/F vs control & 6,19606459 & 1,72497153 &, 001 \\
PC10\% c/F vs control & 2,04226442 & 1,72497153 &, 466 \\
PC10\% s/F vs PC10\% c/F & 4,15380017 & 1,72497153 &, 047 \\
\hline
\end{tabular}

\section{Discusión}

El blanqueamiento dental es uno de los procedimientos estéticos más solicitados en la Odontología Moderna, es un tratamiento conservador pero a pesar de eso se debe tener en cuenta sus diferentes efectos adversos, uno de los cuales se da en la unión sistema adhesivo-esmalte; de la misma manera han aparecido en el mercado agentes blanqueadores que tienen en su composición el ión fluoruro, básicamente para reducir la sensibilidad dentaria, tal como lo demostró Apéstegui M. ${ }^{(7)}$, pero poco se sabe de las alteraciones que podría causar en la unión sistema adhesivo-esmalte.

Los resultados de este estudio demostraron que la fuerza de adhesión de un sistema adhesivo a la superficie de esmalte blanqueado con PC10\% sin flúor (Opalescence) y a otro blanqueado con PC10\% con flúor $0,11 \%$ (Opalescence PF) presentaron ambos una disminución comparándola con un esmalte no blanqueado. Sin embargo se observó que solo la fuerza de adhesión de un sistema adhesivo a la superficie de esmalte blanqueado con $\mathrm{PC} 10 \%$ sin flúor presentó una diferencia significativa con respecto al esmalte no blanqueado, tal como lo demostraron Cavalli V y col. ${ }^{(8)}$ (2004), Giannini M y col.(4) (2006), Silva y col. $^{(9)}$ (2005), Lai S.C.N y col.(10) (2002), quienes usaron el mismo agente blanqueador del estudio, peróxido de carbamida al $10 \%$, pero otro sistema adhesivo, el Single Bond; otras investigaciones también usaron el mismo agente blanqueador del estudio pero 
con sistemas adhesivos como All Bond 2, One-Step y Heliobond vivadent ${ }^{(11,12)}$, también existen estudios que demostraron esta diferencia significativa colocando la resina inmediatamente después del blanqueamiento con PC10\% ${ }^{(13)}$ y con PC15\% ${ }^{(14)}$

Asimismo la fuerza de adhesión de un sistema adhesivo a la superficie de esmalte blanqueado con $\mathrm{PC} 10 \%$ con flúor al $0,11 \%$ es similar a la fuerza de adhesión de un sistema adhesivo a la superficie de esmalte no blanqueado, tal como lo demostraron Giannini $\mathrm{My}$ col. ${ }^{(4)}$ (2006) quienes trataron al esmalte humano con agentes blanqueadores en base a PC $10 \%$, PC10\% con $0,05 \%$ Ca, $\mathrm{PC} 10 \%$ con $0,2 \% \mathrm{Ca}, \mathrm{PC} 10 \%$ con $0,2 \% \mathrm{~F}, \mathrm{PC} 10 \%$ con $0,5 \% \mathrm{~F}$ concluyendo que los agentes blanqueadores en base a PC10\% que contienen en su composición Ca o F pueden preservar la ultima fuerza de tensión después de un régimen de blanqueamiento; sin embargo este resultado difieren con los encontrados por Metz y col ${ }^{(14)}$ (2007) quienes concluyeron que el esmalte blanqueado con PC15\% con flúor reduce significativamente la fuerza de adhesión, pero cuando se coloca la resina compuesta inmediatamente después del blanqueamiento.

Finalmente el estudio demostró que la fuerza de adhesión de un sistema adhesivo a la superficie de esmalte blanqueado con $\mathrm{PC} 10 \%$ con flúor es significativamente mayor comparándolo con la superficie de esmalte blanqueado con PC 10\% sin flúor.

Estos dos últimos resultados del estudio demuestran que la incorporación del ión fluoruro en el agente blanqueador puede preservar la fuerza de adhesión de una resina compuesta a la superficie de esmalte, colocado la resina luego de un periodo de espera de 1 semana después de haber realizado el blanqueamiento, la cual duró dos semanas, a comparación de un agente blanqueador que no contenga flúor en su composición; probablemente esto se deba porque el ión fluoruro al actuar sobre la superficie del esmalte al mismo tiempo que el peroxido produciría un proceso de desmineralización provocado por el peroxido, y un proceso de remineralización provocado por el flúor; este ultimo se da cuando el ión fluoruro ingresa a la estructura del esmalte simulando un sistema de transporte activo y pasivo, luego el ión fluoruro interactúa con los grupos $\mathrm{OH}$ de la hidroxiapatita formando fluorapatita, pero dependiendo de la concentración del flúor no solo se forma fluorapatita sino también fluoruro de calcio; independientemente que se forme fluorapatita o fluoruro de calcio va existir un aumento del gradiente interno de compuestos minerales; por tanto el ión fluoruro incorporado en el agente blanqueador provocaría un proceso de remineralización en el esmalte, tal como lo demostró Gladwell $\mathrm{y} \mathrm{col}^{(3)}$ (2006) quienes concluyeron que la adición del flúor a un sistema blanqueador dental mejora las propiedades de remineralización y que no afecta la eficacia del gel blanqueador.

Por lo tanto al mejorar las propiedades de remineralización en el esmalte que es blanqueado, se produce un aumento significativo de la resistencia del esmalte durante una desmineralización (blanqueamiento) la cual estaría favoreciendo la fuerza de adhesión, debido a que esta se ve afectada cuando existe alteraciones morfológicas en la superficie del esmalte por pérdida de minerales.

Por los estudios revisados numerosos autores han reportado que el blanqueamiento del esmalte con peróxido de carbamida al $10 \%$ con o sin incorporación del flúor provoca alteraciones morfológicas en la superficie del esmalte, perdida de minerales, además de una pérdida de microdureza, los cuales pueden afectar la fuerza de adhesión, una razón para ello se basaría en la teoría estructural, la cual afirma que las alteraciones en la superficie del esmalte que ha sido sometido a un régimen de blanqueamiento puede afectar la fuerza de adhesión disminuyéndola.

\section{Conclusiones}

Bajo las condiciones de este estudio in vitro, después de un régimen de blanqueamiento dentario; la incorporación del flúor en el agente blanqueador preservó la fuerza de adhesión de un sistema adhesivo al esmalte blanqueado comparado con el mismo agente blanqueador sin contener fluor.

\section{Referencias bibliográficas}

1. Alves R. Estética Odontológica. 1ra edición. Sao Paulo-Brasil. Editorial Artes Médicas; 2003

2. Haywood VB. History, safety and effectivencss of current bleaching techniques and application of the nigthguard vital bleaching technique. Quintessence Internacional1992; 23(7): 471- 88

3. Gladwell J, Simmons D, Wright JT. Remineralization potential of a fluoridated carbamide peroxide whitening gel. Journal of esthetic and restorative dentistry. 2006; 18(4):206-12, discussion 212-3

4. Giannini M, Silva A, Cavalli V. Effect of carbamide peroxide-based bleaching agents containing fluoride or calcium on tensile strength of human enamel. J Appl Oral Sci. 2006; 14(2): 82-7

5. Petkova M. Efectos Clínicos y estructurales del blanqueamiento. Odontología Sanmarquina. 2004; 8 (1): 25-9

6. International Organization for Standardization [consultado el 20 de setiembre del 2007] http://www.iso. org/iso/standards_development/ technical_committees/list_of_iso_ technical_committees/isotechnical_ committee.htm?commid=51218

7. Oliveira R, Paes Leme A, Giannini M. Effect of a carbamide Peroxide Bleaching gel containing Calcium or Fluoride on Human enamel surface microhardness. Braz Dent J. 2005; 16(2): 103-6

8. Goo DH, Kwon TY, Nam SH, Kim HJ, Kim KH, Kim YJ. The efficiency of $10 \%$ carbamide peroxide gel on dental enamel. Dental Materials. 2004; 23(4):522-27

9. Metz MJ, Cochran MA, Matis BA, Gonzalez C, Platt JA, Pund MR. Clinical evaluation of $15 \%$ carbamide peroxide on the surface microhardness and shear bond strength of human enamel. Operative Dentistry. 2007; 32(5):427-36

10. Apéstegui Pinto, Marcia. Cambios de hipersensibilidad en dientes vitales durante el tratamiento de blanqueamiento con peróxido de carbamida al 20 por ciento, 3 por ciento de nitrato de potasio y 0.11 ión flúor (Opalescence PF) .Año 2000. Tesis para obtener el título de cirujano dentista

11. Lai SC, bleaching agent effecTay FR, Cheung GS, Mak YF, Carvalho RM, Wei SH, Toledano M, Osorio R, Pashley DH. Reversal of compromised bonding in bleached enamel. Journal of Dental Research. 2002; 81(7):477-81

12. Pinto CF, Oliveira R, Cavalli V, Giannini M. Peroxide ts on enamel surface microhardness roughners and morphology. Brazilian Oral Research. 2004; 18(4):306-11

13. Schwartz R, James B, Summit, J. William Robbins. Fundamentos en odontología Operatoria un logro contemporáneo. 1ra edición. Caracas-Venezuela: Actualidades Médico Odontológicas Latinoamericana; 1999

14. Attin T, Kocabiyik M, Buchalla W, Hanning C, Becker K. Susceptibolity of enamel surfaces to demineralization after application of fluoridated carbamide peroxide gels. Caries Research. 2003; 37(2): 93-9 